\title{
New Strategies for Combining Mindfulness with Integrative Cognitive Behavioral Therapy for the Treatment of Generalized Anxiety Disorder
}

\author{
Lobsang Rapgay · Alexander Bystritsky • \\ Roger E. Dafter · Michelle Spearman
}

Published online: 10 June 2009

(C) The Author(s) 2009. This article is published with open access at Springerlink.com

\begin{abstract}
Generalized anxiety disorder (GAD) severely impacts social functioning, distress levels, and utilization of medical care compared with that of other major psychiatric disorders. Neither pharmacological nor psychotherapy interventions have adequately controlled cardinal symptoms of GAD: pervasive excessive anxiety and uncontrollable worry. Research has established cognitive behavioral therapy (CBT) as the most effective psychotherapy for controlling GAD; however, outcomes remain at only $50 \%$ reduction, with high relapse rates. Mindfulness has been integrated with CBT to treat people suffering from numerous psychiatric disorders, with mindfulness based stress reduction (MBSR) being the most researched. Preliminary evidence supports MBSR's potential for controlling GAD symptoms and key researchers suggest mindfulness practices possess key elements for treating GAD. Classical mindfulness (CM) differs significantly from MBSR and possesses unique potentials for directly targeting process and state GAD symptoms inadequately treated by CBT. This article introduces the theory and practice of CM, its differences from MBSR, and a critical review of MBSR and CBT treatments for GAD. CM strategies designed to complement CBT targeting cardinal GAD symptoms are outlined with a case study illustrating its use.
\end{abstract}

Keywords Classical mindfulness - Generalized anxiety disorder · Mindfulness based stress reduction - Cognitive behavior therapy · Buddhist meditation · Psychotherapy integration

\footnotetext{
L. Rapgay ( $\bowtie)$

Department of Psychiatry, UCLA Resnick Neuropsychiatric Hospital \& Semel Institute for Neuroscience and Human Behavior, P.O. Box 956968, Suite 2200, 300 Medical Plaza, Los Angeles, CA 90095-6968, USA

e-mail: 1rapgay@mednet.ucla.edu
}

A. Bystritsky · R. E. Dafter · M. Spearman

Department of Psychiatry and Bio-Behavioral Sciences, University of California, Los Angeles, CA, USA 


\section{Introduction}

Generalized anxiety disorder (GAD) was regarded for several decades as a residual diagnosis to classify patients not meeting the criteria for other anxiety disorders. In 1980, the Diagnostic Statistical Manual III recognized the condition as a distinct diagnosis (Mennin et al. 2004). The DSM IV TR currently identifies the cardinal symptoms of GAD as excessive anxiety and uncontrollable worry over a number of events or activities, occurring for at least 6 months. Secondary symptoms include at least three of the six following diagnostic criteria: restlessness, being easily fatigued, difficulty concentrating, irritability, muscle tension, and disturbed sleep.

The lifetime prevalence rate of GAD in the United States is estimated to be approximately 4-7\% with current prevalence rates ranging from 1.5-3\% (Blazer et al. 1991). These rates could be as high as 5-8\%, if the international classification of diseases and health related problems (ICD-10) is used as the basis for diagnosis (Ustun and Sartorius 1995). GAD often occurs early in life with twice the number of women suffering from it compared to men. Evidence shows GAD's impact on social functioning, distress levels, and utilization of medical care is equivalent to those of other major psychiatric disorders (Mennin et al. 2004).

Research in the treatment of GAD has primarily focused on the efficacy of pharmacotherapy. More recently, there has been an increasing interest in the efficacy of psychotherapy. Of all the therapies, cognitive behavioral therapy (CBT) has established the most empirical support as an effective treatment for GAD (Gould et al. 2004). The most successful CBT treatment protocols have included motivational therapy, interpersonal psychotherapy, integrative CBT (ICBT) to treat GAD (Baer 2003). However, outcome rates for controlling symptoms are about $50 \%$, with high rates of relapse. While CBT interventions show greater promise than other approaches, more studies are needed to confirm these preliminary findings (Chambless and Gillis 1993).

A number of approaches have integrated features of Buddhist mindfulness practices with CBT to treat a number of psychiatric disorders including GAD (Baer 2003). Western mental health practitioners and researchers have argued that the cultivation of mindfulness may be beneficial for people suffering from psychiatric disorders, who are not interested in adopting Asian or other religious practices (Kabat-Zinn 2000). Mindfulness was conceptualized as being a set of skills that can be learned independently of any spiritual or cultural tradition and then applied to help manage psychiatric symptoms. These approaches have included mindfulness based stress reduction (MBSR) (Kabat-Zinn 1982, 1990, 2003), mindfulness based cognitive therapy (MBCT) (Segal et al. 2002), dialectical behavior therapy (DBT) for borderline personality disorders (Linehan 1993a, b), and acceptance and commitment therapy (ACT) mostly for anxiety and major mood disorders (Hayes et al. 1999).

Mindfulness based stress reduction is the main form of mindfulness currently being studied and practiced by Western researchers and clinicians. There are a number of non-randomized and case studies that demonstrated the efficacy of mindfulness as either a stand alone or complementary intervention in the reduction 
of anxiety symptoms (Kabat-Zinn et al. 1992; Orsillo et al. 2003). However, inadequate evidence exists to date to demonstrate MBSR specifically accounts for reductions of GAD symptoms, since all such studies have lacked standard active control groups (Bishop et al. 2004; Toneatto and Nguyen 2007).

Classical mindfulness (CM) which is based on the original forms of Buddhist mindfulness practices, and differs significantly from MBSR, possesses characteristics with strong potential for directly targeting specific process and state diagnostic features of GAD (Rapgay and Bystritsky 2008). These features of GAD include process symptoms such as uncontrollable, excessive and pervasive worrying, problems with disengaging from threatening stimuli, and state symptoms such as the narrow, and constricted states of attention characteristic of anxiety (Easterbrook 1959). Such automatic anxiety states restrict the range of experience to only threatening stimuli, since patients cannot re-direct their attention to either neutral or pleasant experiences.

The use of mindfulness practices in Western psychology for treating GAD and other psychiatric disorders will be best advanced if a broad range of eastern mindfulness traditions is systematically evaluated within a scientific framework, rather than those that have simply been imported to date. The present article seeks to introduce the theory and practices of CM, its differences from MBSR, and a critical review of MBSR and CBT treatment effects on GAD. Specific CM strategies designed to complement CBT which have strong theoretical potential for targeting cardinal GAD symptoms will be outlined and contrasted to MBSR approaches. A case study will be used to illustrate these specific features and methods of CM. The authors recognize this is likely to be the first attempt to introduce the use of integrating CM with CBT for treating GAD.

We propose that $\mathrm{CM}$ might be a fruitful method for treating GAD that warrants further empirical investigation to determine whether mindfulness approaches can help control features of GAD that have not adequately controlled according to the research studies to date.

\section{A Brief Review of Treating GAD with CBT}

Cognitive behavioral therapy for GAD in the last two decades has progressively evolved through a number of phases of development resulting in better understanding of the triggers and maintenance factors associated with GAD. In the early phase, GAD was treated with Beck's cognitive therapy model originally designed for treating mood and other anxiety disorders. The treatment was a general cognitive approach and non-specific to GAD (Leahy 2004). Borkovec et al. (1983) were among the first to develop a CBT protocol that was specific for treating GAD. They identified excessive, uncontrollable and pervasive worry as a critical symptom of GAD, and they modified the general CBT approach to primarily target this symptom (Borkovec et al. 1998). This included a coping strategy to avoid present emotional distress and future oriented threatening events. However, CBT strategies to reduce and regulate uncontrollable worrying proved to be limited, since anxious patients continued to find other reasons to worry (Dugas 2000). 
Wells (1995) suggested the limits of CBT treatment outcomes for GAD results from only targeting the contents of worry, which he termed "type I worry", and failing to address meta-cognitive beliefs such as "worrying about worry" which he labeled "type II worries." He hypothesized that the "type II worry" was the primary factor in maintaining GAD symptoms. According to Wells, without changing metacognitive beliefs and the behaviors that maintain them, altering "type I worry" has limited outcomes. For example, the use of worry as a coping strategy to avoid threatening thoughts, feelings and activities leads to the development of negative meta-beliefs about worrying itself.

Wells (1995) described how the failure to resolve "type I worry", through planning and problem solving, results in emotional distress and processing difficulties that triggers negative beliefs and appraisals about worrying itself. As a result, there is an increase in threat appraisals, which accentuates anxiety. The patient reverts to "type I worry" as a means to resolve the anxiety thus creating a feedback system that reinforces worrying. Preliminary studies show that Wells' modified CBT, targeting negative meta-cognitive appraisals and beliefs, are effective for treating GAD (Table 1).

Dugas et al. (1998) argue that treating the cause of uncontrollable worrying is more central to successful treatment of GAD, than focusing on the maintenance factors of worrying such as those described by Wells. He proposed that "intolerance of uncertainty" is the core cause of uncontrollable worrying (Dugas et al. 1998). He demonstrated that chronically anxious patients preferred to experience negative outcomes, rather than face uncertainty about future threats, thus creating an illusion of certainty and predictability. These patients also used the avoidance of events resulting in the non-recurrence of threat as evidence that worrying helps to prevent such threats. Dugas et al. (1998) hypothesized that these clinical factors played a critical role in limiting the effect of standard CBT interventions of cognitive restructuring and exposure for GAD even though these interventions helped patients develop more realistic probability estimates of feared outcomes.

The early psychoanalytical literature includes a number of different explanations for anxiety. Starting with Freud's initial presentation of anxiety traced to early sexual repression, and then later his expounding of the signal theory of anxiety, subsequent analysts further elaborated upon the psychodynamics of anxiety. Psychoanalysts such as Horney (1950), Sullivan (1953) and Fromm-Reichmann (1955) presented an interpersonal perspective basis for anxiety. Clinicians from the object relations school such as Fairbairn (1952) stated that anxiety is rooted in the separation conflict of dependency and the fear of being engulfed and experiencing a loss of identity during infancy. Klein (1975) on the other hand, proposed that the roots of anxiety lay in feelings of being persecuted by others resulting from the failure of evoking the primary caretaker and subsequent feelings of incompetence to repair damaged relationships.

In the last two decades, there has been increasing evidence that emotional processing and interpersonal conflict is associated with GAD. Studies show that GAD patients rate interpersonal issues as the main source of their worry (Borkovec et al. 1983). In another study, GAD patients reported significantly more insecure attachment to primary caregivers than those without GAD (Lichtenstein and 
Table 1 Chronology of selected GAD research studies

\begin{tabular}{|c|c|c|c|c|c|}
\hline Date & Author (s) & Description & $n$ & Design & Research outcome \\
\hline 1993 & $\begin{array}{l}\text { Borkovec and } \\
\text { Costello }\end{array}$ & $\begin{array}{l}\text { CT based on Beck's } \\
\text { model for anxiety } \\
\text { disorder }\end{array}$ & 55 & $\begin{array}{l}\text { Pre-post randomized } \\
\text { between groups }\end{array}$ & $\begin{array}{l}\text { Both applied relaxation } \\
\text { (AR) and (CT) } \\
\text { compared. Maintained } \\
\text { gains in both and } \\
\text { highest end-state } \\
\text { functioning for CBT }\end{array}$ \\
\hline 1999 & Newman et al. & $\begin{array}{l}\text { ICBT focused on } \\
\text { emotional and } \\
\text { interpersonal } \\
\text { dysfunction worry } \\
\text { as the primary } \\
\text { issues of GAD }\end{array}$ & 18 & $\begin{array}{l}\text { Within group } \\
\text { pre-post }\end{array}$ & $\begin{array}{l}\text { ES of } 2.87 \text { vs. } 2.16 \\
\text { compared to CBT; } 2.74 \\
\text { vs. } 1.93 \text { at } 1 \text { year } \\
\text { follow-up }\end{array}$ \\
\hline 2000 & $\begin{array}{l}\text { Ost and } \\
\text { Breitholtz }\end{array}$ & $\begin{array}{l}\text { CBT based on worry } \\
\text { and anxiety as the } \\
\text { primary symptoms } \\
\text { of GAD }\end{array}$ & 36 & $\begin{array}{l}\text { Pre-post randomized } \\
\text { between groups, } \\
\text { w/12 months } \\
\text { follow-up }\end{array}$ & $\begin{array}{l}62 \% \text { Significant } \\
\text { improvement in CT } \\
\text { group post-treatment; } \\
56 \% \text { at follow-up }\end{array}$ \\
\hline 2000 & Ladouceur et al. & $\begin{array}{l}\text { CBT based on } \\
\text { intolerance of } \\
\text { uncertainty as the } \\
\text { primary issue for } \\
\text { GAD }\end{array}$ & 26 & $\begin{array}{l}\text { Pre-post randomized } \\
\text { group comparison, } \\
\text { w/6, } 12 \text { months } \\
\text { follow-up }\end{array}$ & $\begin{array}{l}\text { 77\% no longer met GAD } \\
\text { criteria post-treatment, } \\
\text { gains maintained at } \\
\text { both follow-ups }\end{array}$ \\
\hline 2005 & Wells and King & $\begin{array}{l}\text { CBT based on meta- } \\
\text { cognition of worry } \\
\text { as the primary issue } \\
\text { of GAD }\end{array}$ & 10 & $\begin{array}{l}\text { No control, pre-post } \\
6,12 \text { months } \\
\text { follow-up }\end{array}$ & $\begin{array}{l}\text { Significant improvement } \\
\text { (ES ranged from } 1.04 \\
2.78 \text { ). Recovery rates } \\
87.5 \% \text { post-tx; } 75 \% \text { at } \\
6 \text { and } 12 \text { months }\end{array}$ \\
\hline
\end{tabular}

Cassidy 1991). Other studies provided further evidence that GAD patients associated their early trauma experience primarily to friends and family as compared to those without GAD (Molina et al. 1998).

Based on this evidence, clinical research has developed interpersonal, psychodynamic based psychotherapy for GAD, which has forked out into three major directions. The first involves the study of a standardized interpersonally oriented psychodynamic psychotherapy model for GAD (Crits-Christoph et al. 2004), the second is a standardized integrated model of interpersonal-psychodynamic with CBT treatment protocol (Newman et al. 2004), and the third involves an emotional processing based CBT (Moses and Barlow 2006).

The first two models base their understanding of GAD primarily on an interpersonal perspective and trace the origins of the condition to early childhood patterns of attachment which serve as the foundation for the individual's core views of self and others. The authors of the first model hypothesizes that GAD is rooted in early threatening anxiety producing experiences that lead to the formation of basic wishes, expectations, beliefs and feelings about self and others. These wishes are associated with the need for safety, trust, stability and love. However, when the patient connects to these needs the process is disrupted by the activation of fears of being rejected, abandoned, or abused. The fears are so overwhelming that the child not only avoids the threatening triggers of the anxiety but also associates the 
experience of unfulfilled positive needs as distressing as well. This dynamic schema then becomes the template for the patient's future relationships.

In the second model, the authors employ CBT practices to embody the integration of psychodynamic principles and practices. Emotional and interpersonal based sources of danger and associated learned avoidance behavior are identified by conducting a functional analysis, and then systematically targeted for treatment with Socratic questioning, emotional exposure, modeling and skill training strategies. They systematically sequenced the application of interpersonal and CBT therapies in order to facilitate the research process (Newman et al. 2004). Initial studies on CBT co-administered with psychodynamic components resulted in significantly better outcomes as compared to a CBT package alone (Newman et al. 1999). Larger studies are required to confirm these initial findings.

Moses and Barlow (2006) describe a more structured way of addressing emotional disorders such as GAD that is consistent with a CBT model. They suggest emotional dis-regulation not only affects functioning but also is responsible for psychopathology. They refer to studies that show that emotional suppression and maladaptive ways of managing emotions often produces negative consequences. According to the authors a standard emotional regulation approach can be used to treat a range of emotional disorders. They identify three stages of treatment. The first is the restructuring of distorted cognitive appraisals, followed by the identification and treatment of emotional dys-regulation based behavior. The final stage involves targeting emotional avoidance with exposure strategies in order to then facilitate the expression and processing of them (Moses and Barlow 2006).

In fact, from a psychodynamic perspective, there are questions about whether GAD can be treated primarily on the basis of emotional dys-regulation rooted in attachment problems as described in the above models. According to the Alliance of Psychoanalytic Organizations (2006), GAD is a more complex condition that involves signal, separation, moral and annihilation factors depending on the severity of the disorder. Relying entirely on CBT and interpersonal strategies described above may not be adequate to address some of these more underlying factors associated with anxiety.

In summary, there are a number of key considerations in treating GAD that are evident from the extensive CBT studies. First, cognitive, verbal-based, and restructuring strategies appear limited in reducing uncontrollable worry, a cardinal symptom of GAD. Second, an intolerance of uncertainty and avoidance of experiencing anxiety and disengaging from threatening stimuli that maintain the anxiety, are not adequately treated with current treatment strategies. Finally, interpersonal factors related to attachment issues appear important to address in any comprehensive treatment for controlling GAD.

\section{A Review of Current Research and Theoretical Considerations for Using Mindfulness to Treat GAD}

The best known and most researched form of using mindfulness is MBSR which uses a modified form of insight (vispassana) practice from one branch of Buddhism 
to suit the Western mindset developed by its founder (Kabat-Zinn 1990, 2003). A wide body of studies has shown the efficacy of MBSR for a wide range of medical and psychiatric conditions (Bishop et al. 2004; Kabat-Zinn et al. 1998). In the first study on the effects of MBSR on a variety of anxiety disorders including GAD, Kabat-Zinn et al. (1992) conducted an uncontrolled study with 22 patients. They showed significant improvement on several standard measures of anxiety and depression both at post-treatment, and a 3-month follow-up on virtually all the anxiety disorders studied (Kabat-Zinn et al. 1992). A subsequent separate 3-year follow-up study of the same participants showed that treatment gains were maintained (Miller et al. 1995). However, the outcomes for GAD were not as significant as for the other disorders. Furthermore, the studies lacked active control groups; consequently it is difficult to generalize the cause of the treatment outcomes primarily to MBSR due to the constraints of the experimental designs (Toneatto and Nguyen 2007). In fact, when an active control group was used to determine the interaction effects of MBSR on anxiety and depression in a study on psoriasis, no significance was found (Toneatto and Nguyen 2007).

Acceptance and commitment therapy which incorporates a modified version of MBSR with an emphasis on acceptance for anxiety disorders showed significant improvement on a number of outcome measures, but the authors concluded larger studies were needed to verify the efficacy of mindfulness for GAD disorders (Hayes et al. 1999). A recent study combined mindfulness with CBT for treating four GAD patients (Roemer and Orsillo 2002). Results showed significant pre and posttreatment outcomes on the group data. However, the patients individually continued to experience moderate levels of GAD anxiety symptoms post-treatment raising the question as to whether this type of mindfulness added anything clinically to CBT's partial effectiveness reducing GAD symptoms.

While Roemer and Orsillo (2002) based their case treatments on the view that experiential avoidance is a core problem underlying GAD; other critical symptoms which are shown to be central to the successful treatment of GAD were either minimized or ignored. These symptoms included: (1) the excessive, pervasive and uncontrollable nature of worrying (2) the maintaining factors of worrying and anxiety, such as positive and negative beliefs and assumptions about the role of worrying, (3) selective attention to threatening stimuli, (4) the intolerance of ambivalence and uncertainty and, (5) the increasing evidence about the emotional dys-regulation and interpersonal conflict that underlies GAD.

Given the potential of mindfulness for treating GAD, a number of prominent anxiety researchers theorized about underlying processes of GAD that might be impacted by ACT and MSBR strategies to effectively reduce GAD symptoms (Roemer and Orsillo 2002; Wells 2002; Hayes and Feldman 2004). The primary benefits of mindfulness for GAD that the researchers have identified can be subsumed into four broad categories. They are (a) acceptance (b) attention and awareness (c) cognitive change, and (d) present moment experience. The following is a brief critique of the existing literature on mindfulness for the treatment of GAD.

Acceptance has been identified as a primary therapeutic agent of mindfulness with potential for treating GAD. Acceptance is described as a non-judgmental, non-evaluative state of experiential openness of present moment experiences 
(Hayes 2002; Bishop et al. 2004). Patients are simply instructed to adapt this state of openness without any specific training, and it therefore becomes a cognitive or self talk strategy in approaching anxiety. Roemer and Orsillo (2002) combine acceptance and mindfulness integrated into a CBT protocol to target the primary cluster of symptoms of GAD i.e. experiential avoidance. Based on the theory of ACT that the learned habitual attempt by GAD patients to control internal experiences is the major cause of psychopathology, they conceptualize acceptance as an alternative response. Facilitating contact with immediate experiences, including aversive ones, results in replacing habitual biased avoidance patterns, which maintain anxiety. By cultivating a willingness to experience the threat as it is, rather than as either good or bad, patients learn to develop tolerance of threatening events. As greater tolerance develops, the patient is now more amenable to pursing and engaging in valued behavior (Roemer and Orsillo 2002).

However, there is no conclusive evidence that acceptance is able to extinguish fears about future oriented threats. In fact, studies of selective attention to threatening information associated with various anxiety disorders, including GAD, demonstrate that the threat often operates outside awareness (Martin et al. 1991). It is, therefore, very unlikely that acceptance possess mechanisms to adequately access or process aspects of the anxiety that are deep seated beliefs and schemas that are not accessible to conscious recall.

Craske and Hazlett-Stevens (2002) has critiqued the conceptual basis for the use of acceptance strategies for treating GAD. She points out that simple acceptance of threat and danger signals inherent in GAD can conflict with the instinctive need of the individual to use fear and anxiety to protect himself/herself against danger. Craske and Hazlett-Stevens (2002) also raised the point that acceptance could be another subtle form of control. She challenges Roemer and Orsillos' premise that all psychopathology is rooted in trying to control internal experiences arguing that much of the success of CBT in treating anxiety disorders is primarily based on control based strategies.

Roemer and Orsillo (2002) also suggested that mindfulness helps to replace rigid ways of responding with more flexible ones by means of developing attention to cues, responses and contingencies in the present moment. Others speculated that mental flexibility developed through this method and then applied to anxiety experiences can help to reduce patterns of cognitive rigidity associated with anxiety and worry by inhibiting secondary elaborative processing of thoughts and feelings (Wells 2002). However, the proponents do not explain what features of MBSR accomplish these therapeutic tasks. Wells (2002) and Martin (1997) go further and suggest that awareness acquired through mindfulness helps patients detach from the habitual way of responding to negative and threatening stimuli. They suggest that detached awareness helps develop a meta-cognitive mode of seeing thoughts as mental events to be processed, rather than as facts. However, the authors have not explained how learning to detach from habitual experience can help to significantly reduce excessive, pervasive and uncontrollable worrying and anxiety. Given the nature of the worry and anxiety in GAD, it is unlikely that simply learning to observe in a detached way while temporarily providing relief-just like relaxation 
and positive affirmation do in anxiety-is able to undo the entrenched distortions associated with anxiety.

Orsillo et al. (2003), like many others, claimed that present moment experiencing associated with mindfulness helps to reduce anxiety about future oriented threatening events. They implied that training a GAD patient to think about present moment experiences can actually prevent them from thinking about future oriented threatening events by developing flexible ways of responding to environmental contingencies as opposed to more rigid, verbal, rule governed processes. However, they have not explained how focusing on present moment experiences actually stops anxious thoughts and feelings in patients with GAD. In fact, given the nature of anxiety, it is very likely that focusing on present moment experiences and avoiding engaging with future oriented thoughts and experiences could actually result in a negative reinforcement of anxiety.

The above critique about the therapeutic benefits of mindfulness for GAD is designed to highlight the need for further exploration of a range of mindfulness approaches, so that their potential for therapeutic applications might be more fully realized. We therefore want to introduce $\mathrm{CM}$ as an additional set of skills, which can be scientifically evaluated for treating GAD.

The authors present CM not to replace MBSR, or other mindfulness approaches tested in CBT to date, but to build upon them and to stimulate future research to determine which critical elements of mindfulness practices target specific features of GAD symptoms. We will contrast how the practices of CM and MBSR are derived from the historical foundations of Buddhism and then present how CM skill sets have strong theoretical potentials for better controlling a number of GAD symptoms that have not been effectively alleviated by either MBSR or CBT according to basic research to date.

\section{Unique Features of Classical Mindfulness to Treat GAD}

Classical mindfulness practice is based on earliest known teachings of the Buddha and involves the combination of (1) concentration associated with calm abiding (samatha) practice to acquire direct experience, and (2) discriminative analysis associated with insight (vipassana) practice to acquire insight and wisdom. In contrast, MBSR and contemporary mindfulness approaches emphasize more the discriminative observation tradition to acquire insight while excluding specific training in concentration to acquire direct experience.

These differences between CM and MBSR are derived from a consideration of various approaches to mindfulness as it has been defined within a rich and extensive array of historical and contemporary Buddhist literature as well as the many oral traditions of Buddhist meditation practices (Analayo 2006; Bodhi 2006, communications). Within a year after the death of the Buddha in the fifteenth century BC, a split occurred among the various followers of the Buddha. Early Pali texts indicated that mindfulness prior to this split was thought of as combining both insight (vispassana) and calm abiding (samatha) using concentration (Bhikkhu 1999a, b, 2000). The emergent point of view after the spilt began to stress the importance of 
discriminative analysis or insight (vipassana) over calm abiding (samatha) acquired through concentration to the point that mindfulness (sattipathana) became virtually synonymous with discriminative analysis (vipassana) and this point of view has been passed down through the teachings of the proponents. However, this vispassana interpretation of mindfulness is regarded as incomplete according to the leading contemporary scholar/practitioners of Theravada Buddhism, who suggest that the earliest canonical texts emphasize the complementary nature of calm abiding (samatha) acquired through concentration, and insight (vipassana) in the original formulations regarding mindfulness (Bodhi 2000).

Classical mindfulness therefore combines concentration based practices designed to acquire refined, sustained perceptual abilities often referred to as bare attention or direct experience, with practices of detached, discriminative observation to acquire insight into the nature and function of phenomena. To better illustrate the two ways of knowing, take the example of knowing a storm by being in the midst of the storm and experiencing it directly, as compared to knowing the storm by observing it in a detached way from the safety of the living room. Each of the two ways provides their own unique source of acquiring knowledge about experience (Geshe 1992). Rather than relying on just on one of the two, as in the case of insight (vipassana) based mindfulness, combining the two appropriately provides the range and depth of knowledge necessary. Discriminative analytical strategies such as cognitive restructuring or inferential understanding through psychodynamic interpretations to treat anxiety symptoms, while providing valuable information to the patient to know about their pathology, is however not adequate to resolve the problem. As a result, direct experience based strategy of in vivo exposure is used to complement the treatment of GAD. For instance, to help a patient overcome their fear of snakes, both the ways of knowing are absolutely necessary. Discriminative analysis helps the patient to understand how their misappraisals maintain their fear. Direct experience through exposure to the snake for extended period of time helps them to extinguish their fear. The authors argue the need, therefore, to go beyond current forms of mindfulness based on the insight (vipassana) approach, and study classical mindfulness for the range and depth it adds to clinical work.

This brief exposition of traditional formulations of mindfulness grossly oversimplifies the complex historical and contemporary points of view within voluminous Buddhist literature and oral traditions surrounding specific mindfulness meditation practices. However, it serves to provide a context for some of the more widely accepted broad distinctions concerning mindfulness, which forms the basis of differences we are highlighting in CM and MBSR skill sets.

The authors believe that $\mathrm{CM}$ skills training have strong theoretical potential to more effectively alleviate specific GAD symptomatology. For example, one of the main features of GAD is excessive self-verbalization and thinking that contributes towards the maintenance of uncontrollable and excessive worrying (Freeston et al. 1996). CBT self-verbalization and thinking based strategies such as logical based empiricism, exposure and problem solving may inadvertently encourage an individual to continue worrying by finding other probabilities of the threat occurring. Patients who develop skills to sustain engagement with the totality of a target stimulus, while inhibiting the tendency to use global labels to the experience, 
reduce their excessive self-verbalization. The ability to fully engage with the totality of an experience in a sustained way will implicitly inhibit excessive selfverbalization. A number of brain imaging studies confirm that excessive verbalization is a major factor in the maintenance of GAD (Jack et al. 1997). These studies show excessive activation of the left inferior frontal cortex and the speech area of the brain in GAD patients. Studies also indicate that decrease in its activity was brought about by both CBT and pharmacotherapy.

Another feature of GAD that is not directly treated by CBT is the narrow, constricted and rigid state of anxiety that restricts attention to threatening stimuli to the exclusion of neutral and pleasant stimuli. It is very likely that logical empiricism based strategies of CBT are not always successful in significantly reducing worrying and anxiety in GAD patient partly because the strategies are less effective when the state of anxiety is rigid and confines to selective attention to threatening stimuli. Classical mindfulness involves training in awareness, which involves learning to expand the state of mind into a more open, expansive and flexible state in order to develop the ability to contain both maladaptive and adaptive thoughts and feelings. Developing such a skill through repeated training eventually enables the patient to convert the narrow and rigid state of anxiety into a more open and flexible state that is capable of attending to neutral and pleasant stimuli with reduced interference from preceding threatening stimuli.

Research shows that vigilance and problems of disengaging from threatening stimuli is a mechanism of anxiety disorders (Koster et al. 2004). Selective attention to threatening meaning maintains the overestimation of risk and danger that eventually overrides the mental ability of the patient to regulate his or her thoughts and emotional responses. The patient becomes overly sensitive to threat related cues and signals (Matthew and Mackintosh 1998). Training the patient to first fully engage with the threatening stimulus for prolonged periods of time will help to develop increasing familiarity and tolerance of the threat. Once the patient can tolerate the threat, it becomes easier to learn how to let it go of the stimulus with repeated training. The freed up attentional resource is now available for engagement with new, nonthreatening stimuli with minimal interference from the previous threatening stimuli. Being able to sustain full engagement with the totality of the threatening stimulus will help to develop habituation to the threat, which facilitates disengagement from and then orientation and engagement towards, the new non-threatening stimulus.

A final major clinical feature of GAD, experiential avoidance, has not responded fully to conventional CBT exposure. It is likely that habituation based exposure of classical mindfulness may be more appropriate and beneficial. One of the major problems identified with using conventional exposure to target anxiety triggers in GAD is the fact that the anxiety triggers are not only multiple in nature but more importantly many of them are internal and often not easily available to consciousness. Conventional exposure works well in other anxiety disorders such as phobias because the trigger of the fear is a circumscribed and tangible behavior such as heights, flying or spiders. Classical mindfulness provides an alternative form of exposure that seeks to develop habituation of negative and threatening stimuli instead of trying to directly extinguish them. Habituation is developed by repeatedly training to attend to the totality of the primary rather than just the threatening aspect 
of the stimulus while learning to simultaneously be aware of secondary threats in peripheral field of experience without attending to them. Habituation is a behavior acquired through repeated engaging with the primary and secondary threats till the threats no longer evoke the same response as before (Wolpe 1973).

\section{Differences Between Classical Mindfulness and MBSR within a CBT Context}

The efforts to secularize and simplify MBSR by researchers and clinicians has resulted in equating mindfulness with the function of being mindful itself-that is the act of paying attention and being aware of each moment of experience (Roemer and Orsillo 2002). However, understanding mindfulness simply as a state of being mindful of each experience runs the risk of minimizing the traditional complexity of and the potential effectiveness of the practice. It is, therefore, important to understand some additional differences between MBSR based mindfulness and CM skills and practices.

The CBT literature on modern versions of mindfulness makes no clear distinction between attention and awareness, which has led to considerable confusion about the nature of mindfulness (Kabat-Zinn 2000; Bishop et al. 2004). In fact, major researchers such as Bishop, Baer and others have not only failed to provide clear definitions of attention and awareness, but also often used the two interchangeably (Bishop et al. 2004; Baer 2003). Bishop et al. in their effort to define mindfulness describes it as focused attention at one point and at another as an invitation to and awareness of any experience that arises in the field of consciousness (Bishop et al. 2004). Brown and Ryan (2004) rightly raised the question about how it is not possible to focus attention on the breath fully while inviting and being aware of extraneous thoughts, feelings and sensation at the same time.

According to traditional Buddhist literature, attention as a function is the act of focusing single pointedly on a selected object to the exclusion of other experiences for an extended period of time. As a state, it is narrow and focused (Geshe 1992). Awareness as a function is the act of observing, while as a state it is spacious, expansive, and with a containing capacity (Geshe 1992).

Many researchers and clinicians such as Teasdale, Zindel and Wells have defined mindfulness as a meta-cognitive state and function. However, Brown and Ryan (2004) raised questions about whether mindfulness can be regarded as a metacognitive process, that is, a cognition or thought of another thought or experience. They argued that mindfulness is a perceptual process (Brown and Ryan 2004). According to classical Buddhist literature, the initial phase of mindfulness is primarily a perceptual and not a meta-cognitive state. However, contrary to what Brown and Ryan (2004) suggested, classical mindfulness practice at its later phase reinstates meta-cognitive processes in order to encode experience acquired through the first phase of mindfulness. The need for meta-cognitive or detached discriminative observation as an integral part of mindfulness is evidenced in the four foundations of mindfulness mentioned in the Satipathana texts. Since the 4th foundation, which is translated as phenomena, is more subtle and abstract than the preceding foundations, it cannot be known via perceptual or direct experience and is therefore accessed with discriminative processes. 
One of the main differences between MBSR based mindfulness practices and CM is whether acceptance is a feature and function of mindfulness. MBSR based definitions of mindfulness such as proposed by Bishop et al. (2004), attribute acceptance as one of the two central features and function of mindfulness. The concept of acceptance is contrary to the traditional theory and practice of mindfulness since acceptance, as a pre-conception, interferes with the development of direct experience, which is the objective of the first phase of mindfulness. In fact, the centrality of acceptance in mindfulness as a distinct construct separate from attention and awareness has been challenged. Brown and Ryan (2004) in a factor analysis study, showed acceptance does not provide any separate advantage over attention and awareness and appears to be functionally redundant in mindfulness.

In original Buddhism, mindfulness is characterized as the ability to achieve direct experience of an object of attention (Thera 1973). This is the experiencing of either an object or event without any preconceptions of thoughts, attitudes, or emotional tones that interfere with pure, direct experience. Any preconceived values such as acceptance and non-judgmentality of MSBR interferes with the ability to engage with the stark, bare experience of an object. Mindfulness practices enable people to develop bare attention (used here synonymously with direct experience) so that all of these preconceptions are eliminated from the experiencing of an object. In order to experience the "bare" sound of a police car, the sound must be stripped of its ascribed meaning of threat, so that awareness becomes free from any and all preconceptions. Similarly, in order to experience a negative experience such as an aversive sensation, the sensation must be stripped of all projections in order to experience it as it is. Thus, the intention or cognitive set to classify the sensation within a framework of acceptance must also be suspended, since it interferes with the direct experience of the sensation as it is in the moment.

Openness to whatever experiences arise in consciousness as a function of MBSR is also in contrast to original instructions for classical mindfulness training (Bhikkhu 1999a, b, 2000). Bhikkhu (2002) states that according to classical mindfulness literature an ability to be open to whatever experience occurs without judgments and reactivity is achieved much later in mindfulness training and, therefore not during the initial training phase. The patient should be aware of both adaptive and maladaptive thoughts, feelings and behavior. There is also the danger that openness and curiosity can actually obstruct the development of attention and awareness skills. Since the goal of mindfulness is to learn how to reduce sensitivity to external stimuli in order to access internal ones that are often not available to conscious awareness, encouraging curiosity and inviting experiences is likely to increase preoccupation with contents of thoughts and feelings, the very thing that one is trying to overcome in mindfulness.

Some researchers such as Baer (2003) and others suggest that mindfulness is a state of non-striving-without any specific objective. They argue the therapeutic benefits of such an approach as an alternative to the habitual process and demands of working towards goals (Baer 2003). However, classical mindfulness has specific goals associated with its various phases of practice (Bodhi 2006, communications). All Buddhist teachings, including those on mindfulness, have the explicit overall goal of eliminating maladaptive thoughts, feelings and behavior in order to replace 
them with positive ones. Consequently, every major aspect of mindfulness training involves the identification of specific goals, means to achieve them and methods to assess the outcomes.

Mindfulness based stress reduction and other forms of mindfulness practices highlight the centrality of present moment experiences. Roemer and Orsillo (2002) even go to the extent of suggesting that past memories and future fears should be avoided during mindfulness. Classical mindfulness on the other hand involves mindfulness of present, past and future experiences. While the breath is used as the anchor to develop attention and awareness, all past, present and future experiences are treated as distractions that the patient identifies and labels and then returns to the attention and awareness of the breath. In classical mindfulness, being in the present moment is simply understood as being aware of whatever contents arise during attention and awareness on the breath. In fact, trying to suppress past and future experiences could act as a negative reinforcement of anxiety, the very problem that the authors claim they are seeking to eliminate in the treatment of anxiety.

Some modern versions of mindfulness have further suggested that mindfulness facilitates emotional experience and regulation. Hayes and Feldman (2004) see mindfulness as an emotional balancing technique to help regulate mood, and increase clarity of affect. They quote teachers like Chodron (2001) to describe how mindfulness can be used to feel the full force of an emotion as a way to overcome it. Marsha Linehan, Sona Dimidjian as well as Kabat-Zinn (2003) also imply that mindfulness practice involve affective related practices such as compassion. However, in Buddhist literature exploring affective states such as compassion is an entirely separate practice introduced only after developing skills in mindfulness practices (Pabongka 1997). Using mindfulness to experience powerful emotions in their full force is contraindicated for the simple reason that doing so would obstruct the development of sustained attention and awareness, which is the primary goal of mindfulness. Rather, in classical mindfulness when an affect arises, it is observed and immediately labeled as a feeling and then the patient returns to the primary task of experiencing the breath. The objective is to observe the arising of the emotion in order instantly identify what triggered the emotion as well as notice what consequences follow. The experiential insight thus gained into the triggers and consequences of the emotion helps to regulate habitual reactive patterns and increase more adaptive ways of responding.

\section{Specific CM Skills for Treating GAD}

There are two objectives associated with CM skill training for treating GAD: (1) to achieve a level of sustained, detailed, non-conceptual divided attention and awareness (also known as bare attention or direct experience), and (2) develop the ability to carry out experiential based insight based on the way of experiencing as described in (1). These two objectives clearly imply that there are two major stages of mindfulness practice. The first stage is training in sustaining, detailed, nonconceptual divided attention and awareness which needs to be distinguished as significantly different from MBSR practice of mindfulness. The second stage 
involves the reinstatement of gradual application of discriminative processes informed by direct experience in order to enrich the process of knowing.

In order to achieve the first objective, the patient must learn and acquire an ability to (a) focus in a sustained way on a target object (such as the breath), as well as (b) intimately experience details of the features and functions of the target in its totality and do so (c) by implicitly inhibiting the use of verbal and conceptual processes. In addition, the first phase of mindfulness involves additional processes of monitoring, disengaging from stimuli and open-ended exposure. When the patient acquires a basic level of skill in these various processes, then the patient is ready to pursue the second objective. The second objective involves acquiring proficiency in the application of experiential insight. Experiential insight involves learning how to carry out discriminative analysis in a more informed and objective way by relying upon the direct experience information from the first phase of mindfulness to analyze maladaptive and adaptive thoughts, feelings and behavior.

In order to apply mindfulness to target specific symptoms of GAD, the senior author recommends the clinical application of mindfulness in the form of specific skills that are acquired by mastering the seven processes associated with mindfulness practice. The author suggests that the seven processes are an inherent aspect of and are not distinct components from mindfulness.

\section{Seven Processes Associated with Mindfulness Practice}

\section{Non-Conceptual Awareness and Attention}

The two are developed separately and generally attention is developed first and then awareness. However, in psychopathology, it is recommended to develop awareness first. Attention training involves training in sustaining focus attention on a single object to the exclusion of everything else. The goal is to gradually reduce distractions to other stimuli while increasing the ability to focus on the target object for prolonged periods of time. Awareness involves experiencing the expanded, spacious, containing capacity of consciousness. The clinical objective of awareness is to reduce the habitual urge to expel threatening stimuli in order to avoid their experience since repeated expelling of such stimuli results in weakening ego functioning.

The skill of containment acquired through awareness training is applied as the first phase of treatment to convert and expand the narrow, constricted and rigid anxious state into an open and flexible one. The patient breaks up the undifferentiated components of anxiety into its various components, which are then localized in the peripheral field of awareness. Through awareness training, the patient learns to contain the differentiated aspects of anxiety which enables the patient to reduce the habitual urge to expel them.

Having acquired some proficiency in containment, the patient can now move to the next clinical process of inhibiting cognitive biases, which are the basis of misappraisals of stimuli using attention training to facilitate the process. The first strategy is to reduce the habitual tendency to rely on first impressions of an 
experience to define the meaning of a stimuli. The first impression process is often outside awareness and determined by past memories, which bias the experience of the present stimulus. Sustained attention that is fully engaged with the totality of the stimulus helps a patient to be completely immersed in the present moment experience and thus eliminates the first impression processes. The second factor that contributes towards cognitive bias is the use of global labels subsequent to defining a stimulus based on first impressions. The global label consolidates the meaning of the experience by categorizing it. For instance, categorizing a constricting sensation in the chest as "suffocation" triggers a range of meaning such as the patient nearly choking to death as a child and potentially superimposed by images of suffocation, for example from movies that the patient has seen about people suffocating in excruciating pain from poisonous gas. As a result, these associations define the meaning of the constriction in the chest into an exaggerated level of danger and prevent the patient from discovering that the constriction in the chest is really a function of tightening of the chest muscles. Sustained attention on the totality of moment-to-moment sensation in a non-evaluative way helps to discover that the constriction is really a series of varying, discrete units of sensations. As the patient immerses into each of the varying discrete sensations from moment to moment, the ascribed meaning to the sensation as "suffocation" is suppressed resulting in many patients reporting relief from the constriction.

\section{Non-Conceptual Sustained and Detailed Divided Attention and Awareness}

When the patient acquires the ability to induce containment and reduce cognitive bias by decreasing reliance on first impressions and global label as a basis of ascribing meaning to an experience, the patient now attempts in integrate the two into a single experience. The process involves combining attention and awareness into a single practice in order to develop the mental skill of focusing on a primary object while being secondarily aware of the background landscape. The skill helps the patient to experience stimuli in relation to their environment and thus reduce the tendency to selectively focus on a particular aspect to the exclusion of everything else. An anxious patient tends to zoom in on the thought "I will fail" to the exclusion of everything that is occurring in his or her present environment. As a result, the patient is held hostage to his or her anxious thought.

To facilitate mastery of the integrated practice, the senior author recommends the division of attention and awareness into an $80-20 \%$ ratio with $80 \%$ of the mental activity involving focused attention on the primary object and $20 \%$ on awareness of the peripheral field of experience/environment surrounding the primary object.

\section{Introspective Awareness}

During the practice of divided attention and awareness training, the patient observes from time to time whether he or she is engaged fully with the primary object or whether he or she is lost in distractions. However, it is important not to activate introspection too frequently since doing so will obstruct the development of divided 
attention and awareness. Training in self-awareness helps patient to be able to observe themselves and their reactivity and thus able to control them better.

\section{Labeling}

Once the patient is aware that he or she is distracted, the patient engages in labeling the distraction. However, if the contents of the distraction are labeled it is likely to increase preoccupation with the content by generating other associative thoughts and feelings. Labeling of the mental process that generates the content helps to reduce the danger of preoccupation with contents, which tend to maintain symptoms of GAD.

\section{Open Ended Exposure}

With increased capacity to contain the urge to expel threatening stimuli, and reduce cognitive biases as the basis of defining the meaning of the stimulus, the patient focuses on learning how to tolerate negative and threatening stimuli. This is accomplished not by trying to extinguish them, but rather by increasing the power of fully engaging with the totality of a primary stimulus while decreasing interference from the presence of secondary threatening distracters. Tolerance refers to habituation rather than extinction. Craske and Hazlett-Stevens (2002) differentiates between the two by stating that extinction involves the elimination of the fear via repeated and prolonged exposure to the trigger of the fear, while habituation involves learning how to develop tolerance of the overall threatening context without trying to eliminate the associated fear directly.

\section{Habituation}

When the patient habituates to the anxiety and its contextual basis, the patient is now ready to employ the power of sustained engagement with the totality of the stimulus in a non-verbal way. This facilitates disengagement from the threatening stimulus without the threatening stimulus interfering with the orientation and more importantly the full engagement with the next stimulus, particularly pleasant ones. The idea is that the more engaged in a sustained way the patient is in the totality of the primary, threatening stimulus, the more the patient is able to disengage from the stimulus and divert its attentional resources completely to the new stimulus without any of the attentional resources still directed at the threatening stimulus. Research shows that difficulty with disengaging from threatening stimuli is a potential mechanism, which maintains anxiety systems even after cognitive restructuring and exposure therapy. Therefore, using a strategy that directly trains patients to learn how to disengage fully may contribute significantly in improving the outcome of CBT for GAD.

\section{Experiential Insight}

Once the patient is able to apply direct experience strategies described above and replace the habitual way of experiencing with this new way of perceiving anxiety, 
the patient now reinstates discriminative observation. In order to organize what has been learned from the new way of perceiving anxiety and into structures and systems to apply in real time to deal with anxiety. Refined discriminative observation with notation requires training in observation. The level of observation skills will vary with patients, but generally a level equivalent to that of a scientist who studies wild life is necessary. It is important to be able to observe in a discriminative way the mental events that are unfolding without drawing conclusions too quickly. Only when the mental processes have unfolded in a similar way several times can a conclusion and insight be derived. The urge to control events by drawing conclusions instantly prevents deriving the full knowledge that refined discriminative observation is capable of providing.

\section{The Integration of CM with ICBT: An Illustrative Case Study}

In seeking to integrate classical mindfulness with integrative CBT (CMICBT), the main attempt has been to avoid duplication of treatment, and apply strategies from each of the three varying interventions, i.e. CM, ICPT, and psychodynamic therapy, which are more appropriate and effective for targeting a particular symptom. The following case history seeks to illustrate the practical ways to apply CMICBT in 15 sessions.

\section{Participant}

The patient is a divorced Caucasian woman in her mid thirties living with and working for her mother. Her father died about 2 years ago, and the patient claimed she had finally recovered from his death. She recalled that in her short marriage, her husband was verbally and emotionally abusive towards her; however, she could never muster the courage to leave him. Eventually, he left her. As a child, she remembered being anxious and always trying to take care of other family members.

Presenting problems included complaints of uncontrollable, excessive worry daily, "every hour," regarding a number of present and future concerns with underlying emotions of anger, frustration, resentment, guilt and sadness. She reported this worrying was beneficial, because it made her feel she could better prepare for these situations. She also felt little control over worries in response to her long-standing conflicts with her mother. These anxieties were often accompanied by additional symptoms of restlessness, feelings of being on edge, difficulty concentrating, increased irritability, muscle tension and breathlessness. Such distress interfered with attending work, performing at work, socializing and carrying out many daily activities. She had a tendency to procrastinate, and difficulty with making decisions as well as sticking to them. No other anxiety or psychiatric disorders were present and no medical factors contributed to anxiety. A history of major depression existed especially during college, but had been treated successfully with medication and psychotherapy. 


\section{Treatment Protocol}

\section{Step I (Sessions 1-3)}

The patient contracted for 15 sessions of CMICBT including regular compliance with daily assignments and completion of self-monitoring sheets. The patient's chief assignment was the daily $30 \mathrm{~min}$ of practice of the seven classical mindfulness skills, 6 days a week. The patient's goal was to progressively develop each of the seven skills sequentially in daily practice, during the first 6 weeks.

The patient was taught to synchronize her inhalation and exhalation with the movement of her abdomen and to master the process, whereby she could regulate her breathing with the movement of her abdomen rather than through conscious mental control (Table 2). When the breath became slower and finer to the point where the patient did not have to consciously think about it, she was then taught to immerse herself into the sensation of the breath to the point where she could sense the variations in her breathing. This was to occur not only during exhalation and inhalation but also during the beginning, the middle and end of each of the two breaths. The skill of letting her breathing occur spontaneously and utilize the attentional resources to immerse herself into the breath, helped the patient develop control without consciously seeking to do so. Developing control skills without making an effort enabled the patient to not invest in fighting or fleeing from her physical tensions activated by her anxiety.

\section{Assessment}

Assessment included the administration of the mini-psychiatric diagnostic questionnaire with a comprehensive 90 min clinical interview as well as the Beck's anxiety inventory (BAI) and Beck's depressive inventory (BDI) to be administered at the end of treatment and used for evaluating treatment outcomes. The pretreatment BAI score was 44 and BDI was 16.

Assessment also included regularly monitoring the severity, frequency and duration of symptoms. She was instructed to practice classical mindfulness $20 \mathrm{~min}$ daily and the remaining $10 \mathrm{~min}$ to complete the self monitoring sheet of the severity, duration and frequency of her symptoms and to measure the effectiveness of the mindfulness based skills and CBT strategies in reducing anxiety symptoms.

Table 2 Step I (sessions 1-3)

\begin{tabular}{ll}
\hline Intervention strategy & Description \\
\hline Assessment & $\begin{array}{c}\text { Mini-psychiatric diagnostic questionnaire, Beck's anxiety inventory and Beck's } \\
\text { depressive inventory } \\
\text { The psycho-education of the patient involves information about the triggers and } \\
\text { maintaining factors of her anxiety and how classical mindfulness combined } \\
\text { with integrative cognitive behavioral therapy helps to treat GAD }\end{array}$ \\
$\begin{array}{c}\text { Psycho-physiological } \\
\text { regulation }\end{array}$ & $\begin{array}{c}\text { Involves the use of the preliminary practice of classical mindfulness to reduce } \\
\text { the physical tension and arousal associated with anxiety }\end{array}$ \\
\hline
\end{tabular}


Psycho-Education and Psycho-Physiological Regulation

1. Psycho-education involved orienting the patient to the potential cause, triggers, maintenance factors, and reasons why standard treatment do not work, and providing justification for how and why classical mindfulness helps to increase the effectiveness of ICBT for GAD.

2. After psycho-education, the patient received the initial instructions for phase I of treatment. This involved training in synchronized breathing with the movement of the abdomen for regulating physiological arousal associated with anxiety. By being able to regulate breathing with the movement of the abdomen rather than through conscious control, the patient reported a gradual reduction in arousal. It appeared that synchronized breathing not only achieved the same results as progressive muscle relaxation (PMR), but may have required less time.

\section{Step II (Sessions 3-7)}

Research has shown that restructuring of cognitive distortions that underlie GAD does not necessarily lead to significant reduction in uncontrollable worrying and anxiety (Table 3). The authors hypothesized that trying to restructure the maladaptive, anxiety related thoughts, assumptions and beliefs, without first converting the associated constricted and rigid state of consciousness into a more open and adaptive one, prevents a patient from learning how to initiate voluntary redirecting of attention to neutral and positive information. Since by definition, anxiety of GAD results from reflexively orienting the patient towards threatening stimuli triggers, and its sequelae of excessive, uncontrollable worry. An initial modicum of voluntary control of attentional processes must first be restored, before cognitive restructuring and other strategies can be initiated in GAD. This was achieved by the patient training to achieve the seven processes of mindfulness and then applying them to GAD processes in steps II and III.

For example, the patient first learned to recapture some voluntary control of attentional processes. This resulted from learning the key skill of containing threatening stimuli without resorting to the automatic pattern of expelling them instantly. This containment skill consisted of first connecting to the containing capacity within by generating an "internal sense of spaciousness." This was learned in practice sessions by focusing attention either on the sensory experience of the external space of the room or within a circle, while in a calm state of mind. Then the patient practiced internalizing this sense of spaciousness to be within the mind itself.

Once this containment skill was well practiced, it could be applied to anxiety experiences. Generating a sense of spaciousness during anxiety enabled the patient to disrupt the narrow, rigid state of anxiety into the creation of an open and flexible state of consciousness. The patient was then able to engage with the primary anxiety provoking stimuli without expelling it, disrupting the GAD attentional processes of being automatically directed towards the threat. Therefore, the patient had greater voluntary attentional resources, which she could make available towards other 
Table 3 Step II (sessions 3-7): sequential CM and CBT procedures for treating pervasive, excessive and uncontrollable worrying and anxiety

Intervention strategy Description

A. Classical mindfulness based clinical strategies

B. Cognitive and behavioral therapeutic strategies
1. Three mindfulness based clinical strategies associated with the skill of containment target the habitual tendency of the patient to expel negative and threatening experiences

a. Strategy to expand narrow, and rigid states of anxiety into an open and flexible state of mind

b. Strategy to organize constituents of anxiety in the field of awareness

c. Strategy to resolve the disparity of living in the future as compared to being in the present moment

2. Three mindfulness based clinical strategies associated with inhibition of cognitive biases to reduce from ascribing negative and threatening meaning to experiences

a. Strategy to implicitly inhibit excessive self-verbalization and thinking

b. Strategy to implicitly inhibit reactivity

c. Strategy to dismantle global labeling

3. Two mindfulness based clinical strategies associated with monitoring and labeling

a. Identify cues and triggers of threatening stimuli

b. Shifting from content to process

1. Cognitive restructuring to reduce overestimation of risks and catastrophic thinking

2. Worry time and worry free zone to reduce frequency of uncontrollable worrying and anxiety

aspects of the primary threatening stimulus. In the course of several practice sessions, the patient learned that excessive worrying with the contents of the distractive thoughts and feelings resulted in increasing preoccupation with them and shattered the open, flexible and expansive state of mind she had achieved. This loss of spaciousness resulted in becoming fixated on the trigger event again with a reverberating of secondary global meanings of threat.

Having developed the initial capacity to contain threatening stimuli without resorting to the habitual pattern of expelling them instantly, the patient took the second step of repeated practicing, to further strengthen the containment skill. Once strengthened, she had the ability to inhibit the tendency to form evaluative and reactive responses such as global labels to the primary threatening stimuli associated with excessive worry. The patient learned through further practice to contain all anxiety related forms of distractions (primary threat and automatically triggered secondary excessive worry) as events in a spaciousness field of awareness. The patient began to discover that the anxiety, distractions, and worry occurred within a spacious field and then arose and disappeared on their own without further effort. Through repeated training of directing attentional resources to other features and functions of the stimulus, rather than being caught in the automatic selective 
attention to its threatening aspects, the patient was able to have a direct experience of the stimulus, since it was stripped of its threatening aspects and global labels.

Once the patient began to effectively apply the mindfulness skills of containment that reduced these cognitive biases and restored some voluntary control of attention, she was then introduced to CBT restructuring strategies of the primary cognitive distortions associated with GAD. The strategies involved evidence for and against the likelihood of the feared events to occur followed by a cost and benefit analysis to determine alternative, realistic ways of thinking, as compared to the old way of appraising future threats.

Cognitive restructuring of the overestimation of risks and catastrophic thinking was further consolidated with behavioral strategies of setting up specific worry time and worry free zones in order to help the patient develop increasing control over uncontrollable worrying and anxiety.

\section{Step III (Sessions 7-10)}

Since CBT based exposure has shown to be limited for GAD though it is highly effective for other anxiety disorders, mindfulness based open ended exposure was used to help the patient to develop habituation of both the anxiety and its triggers (Table 4). Consistent with the pervasive nature of anxiety and worrying in GAD, mindfulness involves open ended exposure to varying types of anxiety and their triggers unlike conventional CBT exposure which targets a single, tangible behavior in order to extinct the fear completely. Consequently, in mindfulness exposure, the patient was trained to develop habituation of the presence of multiple threats by repeatedly training the patient to allocate $80 \%$ of their attentional resources to the totality of the primary threatening stimulus associated with the anxious affect, and $20 \%$ of their resources to the secondary threats in the peripheral field of awareness.

The patient was repeatedly exposed to thoughts and feelings that are both the triggers of the anxiety as well as those that involve avoidance of anxiety. By allowing thoughts and feelings to arise on their own without the pressure to engage in exposure to them for a prescribed period of time, provided the patient the freedom to eventually develop familiarity and tolerance of the threatening stimuli. As the

Table 4 Step III (sessions 7-10): open ended exposure, behavioral experimentation and problem solving

\begin{tabular}{ll}
\hline Intervention strategy & Description \\
\hline $\begin{array}{l}\text { A. Classical mindfulness } \\
\text { based strategies }\end{array}$ & $\begin{array}{r}\text { 1. Two mindfulness based strategies associated with the skill of tolerance } \\
\text { of negative and threatening stimuli } \\
\text { a. Increase tolerance of threatening stimuli associated with anxiety } \\
\text { b. Increase ability to carry out perceptual and cognitive tasks in the } \\
\text { presence of multiple threatening stimuli }\end{array}$ \\
$\begin{array}{ll}\text { B. Cognitive behavioral } \\
\text { therapy }\end{array}$ & $\begin{array}{l}\text { Behavioral experimentation to test ability to be present and enjoy } \\
\text { positive activities }\end{array}$ \\
\hline
\end{tabular}

The above procedures were used to reduce cognitive, affective and behavioral avoidance of negative and threatening thoughts, feelings, sensations and behavior; in particular this procedure is used to reduce negative and threatening affect 
fear of the threat decreases, the avoidance behavior also diminished. In fact, habituation serves the ultimate function of response prevention.

After the patient developed greater habituation of threatening stimuli, the patient was then trained in the mindfulness skill of disengaging from threatening stimuli rapidly, making use of her voluntary attentional resources. This was by means of repeated training in engaging in the threatening stimulus and then disengaging from it by rapidly orienting and engaging with neutral and pleasant stimuli. The patient was able to reduce vigilance towards threatening stimuli by developing the skill to engage with the totality of the stimulus for extended periods of time. The decreased vigilance helped the patient to disengage from the stimulus, a process which was further facilitated by redirecting attention towards the next new experience. For instance, when the patient could disengage rapidly from the anxious thoughts of a conflict with her mother, she could orient herself more readily to a more realistic and positive thought about her fears. When the patient was able to disengage rapidly from threatening stimuli, the patient was then recommended to behavioral experimentations to test her deep seated fears about specific situations.

\section{Step IV (Sessions 10-14)}

The next five sessions were devoted to the patient's identifying the unfulfilled need from the other, the actual or perceived response of the other, and the response of the self to the other (Table 5). The first step involved helping the patient identify the primary emotion of intense repressed anger in which she wanted to scream at her mother and physically shake her to shut up. Recreating and reliving her past angry situations and the context, in which they occurred, helped her identify the depth of her anger, the resistance, and the complex feelings of fear and guilt it activated. In exploring the underlying source of her anxiety, she experienced a mother who was demanding and critical, initially she was disappointed but quickly that disappointment turned into anger and resentment. The feelings of resentment and anger in turn activated feelings of guilt and fear of retaliation from her mother.

Table 5 Step IV (sessions 10-14): resolving underlying emotional and interpersonal conflict-the source of intolerance of uncertainty

Intervention strategy Description

A. Psychodynamic based intervention

B. Classical mindfulness based strategy
1. Identifying the unfulfilled need from the other, the actual or perceived response of the other, and the response of the self to the other

2. The underlying ambivalence and uncertainty

3. Clinical strategy of experiencing change as the basis of increasing tolerance of ambivalence and uncertainty

a. How the meaning ascribed to triggers determine subsequent reaction and consequent behavior

b. How mental experiences arise, abide and disappear on their own when cognitive biases are inhibited 
The ambivalence of her relationship was maintained by the fact that there was a part of her that deeply wanted her mother to accept her unconditionally as evidenced by her unfailing efforts to do everything to please her mother. However, her inability to resolve her ambivalence between her negativity towards the bad mother and her as the angry, resentful child and her desire for a good mother and her as the perfect child made her intolerant of any ambivalence and uncertainty about future oriented threatening situations. As a result, she could never live in the present moment of experience as she constantly maintained a state of vigilance about future oriented threats. Nor could she fully take advantage of the various strategies to manage her fears because she lived in the fantasy that there existed a future free from all forms of threat.

The patient was educated about the role of the intolerance of ambivalence and uncertainty associated with her interpersonal conflicts. The patient was then introduced to mindfulness based experiential insight strategies designed to help recognize that mental experiences are constantly subjected to change from moment to moment as a way to help her become more tolerant of ambivalence and uncertainty. Consequently, change was not the problem per se but rather how she perceived, appraised and reacted to change was the underlying factor.

Through experiential insight into how the perception and appraisal of a trigger of anxiety determined the subsequent reaction and consequent behavior, the patient became aware of how changing perception and appraisals of triggers of anxiety could result in reduction of her reactions and subsequent behaviors. Having recognized that the meaning ascribed to an event is the basis of anxiety, the patient now also recognized that if no such meaning was actively ascribed, and the event was experienced as an occurrence in the field of awareness, it dissolved into the spaciousness on its own.

\section{Step V(Session 15): Relapse Prevention}

At the end of session 15, her post-treatment BAI score was 17 with a BDI score of 10. The patient also reported significant improvement in GAD symptoms and increased ability to regulate them, though some problems remained in a number of situations. For example, there were less triggers for her, so anxiety reactions were fewer, and she felt confident enough to live separately from her mother, expressing the hope of finding a new job, and relationship. She reported reacting with much less intense physical tension and somatic symptoms to anxious thoughts and concerns, when they did occur. She was also able to prevent uncontrolled worrying most of the time by applying the strategies she had learned. Interpersonally, she could better regulate her reactions to her mother and thus verbalize not only her concerns but needs to her as well.

For relapse prevention, she was encouraged to recognize that anxiety would still persist but now that she had tools to much more effectively cope with it rather than live in constant dread. She was also reminded that during major stressors she should anticipate an increased degree of distress and disruption and the appropriate strategies were systematically outlined for her that she could use for anxiety regulation. 


\section{Discussion}

We have introduced the basic definition and functions of $\mathrm{CM}$ in order to show certain differences with current CBT versions of mindfulness such as MBSR for the treatment of GAD. CM incorporates the two ways of knowing through the integration of (1) concentration acquired through calm abiding (samatha) and (2) insight (vipassana) that we suggest may provides a more comprehensive approach to treat GAD, since MBSR is based on insight (vipassana) training alone.

The authors presents CM not to replace MBSR, or other mindfulness approaches tested in CBT to date, but to build upon them and to stimulate further research to determine which critical elements of mindfulness practices most effectively target specific features of GAD symptoms. Our goal is to engender a broader appreciation for some of the sophisticated theories of perception and cognition which permeates ancient and contemporary Buddhist scholarly and practice mindfulness traditions, so that this wealth of knowledge can be effectively translated and utilized within Western clinical practice for treating GAD.

GAD includes process symptoms such as uncontrollable, excessive and pervasive worrying, problems with disengaging from threatening stimuli, and state symptoms such as the narrow, and constricted states of attention characteristic of anxiety that have not been effectively controlled according to research to date. We have proposed that $\mathrm{CM}$ skills theoretically may have strong potential for alleviating these symptoms. We have provided a detailed account of comparing how MBSR and other mindfulness based introduced into CBT to date contrast with that of $\mathrm{CM}$ in terms of historical foundations, theory, practice and different practical training approaches that might be utilized in CM protocols integrated with CBT for GAD.

The authors recognize this is a preliminary attempt to introduce integrating CM with ICBT for treating GAD with an illustrative single case history. The senior author is currently developing a standardized and manualized protocol in order to conduct the first set of feasibility studies in the application of CM for GAD and OCD at UCLA.

The final verdict of mindfulness for effectively treating GAD will be based upon well controlled CBT outcome research as well as through other investigations such as brain imaging studies with functional MRIs and other neuroscience methods, when subjects are compared after being trained in different mindfulness skills sets. We are aware that the translation of these varied mindfulness practices into modern CBT clinical practices is an ongoing process of arduous study and precise research, in order to fully and accurately capture their full potentials for treating GAD.

Acknowledgments Funding was provided in part by grants from Saban Family Foundation and the Michael E. Tennenbaum Family Fund.

Open Access This article is distributed under the terms of the Creative Commons Attribution Noncommercial License which permits any noncommercial use, distribution, and reproduction in any medium, provided the original author(s) and source are credited. 


\section{References}

Alliance of Psychoanalytic Organizations. (2006). Psychodynamic diagnostic manual (PDM). Silver Springs, MD: Psychodynamic Diagnostic Manual.

Analayo, V. (2006). Satipatthana: The direct path to realization. Birmingham, UK: Windhorse Publications.

Baer, R. A. (2003). Mindfulness training as a clinical intervention: A conceptual and empirical review. Clinical Psychology: Science and Practice, 10(2), 125-143.

Bhikkhu, T. (1999a). The path of concentration and mindfulness. In Noble strategy: Essays on the Buddhist path. Valley Center, CA: Metta Forest Monastery.

Bhikkhu, T. (1999b). One tool among many: The place of vipasana in Buddhist practice. In Noble strategy: Essays on the Buddhist path. Valley Center, CA: Metta Forest Monastery.

Bhikkhu, T. (2000). Maha-satipatthana sutta: The great frames of reference, DN 22. In The connected discourses of the Buddha. Somerville, MA: Wisdom Publications.

Bhikkhu, T. (2002). The Agendas of Mindfulness. Access to Insight Edition 2002.

Bishop, S. R., Lau, M., Shapiro, S., Carlson, L., Anderson, N. D., et al. (2004). Mindfulness: A proposed operational definition. Clinical Psychology: Science and Practice, 11(3), 230-241.

Blazer, D. G., Hughes, D., George, L. K., Swartz, M., \& Boyer, R. (1991). Generalized anxiety disorder. In L. N. Robins \& D. A. Regier (Eds.), Psychiatric disorders in America (pp. 180-203). New York: Free Press.

Bodhi, V. B. (2000). The connected discourses of the Buddha: A translation of the Samyuta Nikayas. Somerville, MA: Wisdom Publications.

Bodhi, V. B. (2006). The nature of mindfulness and its role in Buddhist meditation. A correspondence between Alan Wallace and Venerable Bhikkhu Bodhi.

Borkovec, T. D., \& Costello, E. (1993). Efficacy of applied relaxation and cognitive-behavioral therapy in the treatment of generalized anxiety disorder. Journal of Consulting and Clinical Psychology, 61, 611-619.

Borkovec, T. D., Ray, W. J., \& Stober, J. (1998). Worry: A cognitive phenomenon intimately linked to affective, physiological, and interpersonal behavioral processes. Cognitive Therapy and Research, 22, 561-576.

Borkovec, T. D., Robinson, E., Pruzinsky, T., \& DePree, J. A. (1983). Preliminary exploration of worry: Some characteristics and processes. Behavior Research and Therapy, 21, 9-16.

Brown, K. W., \& Ryan, R. M. (2004). Perils and promise in defining and measuring mindfulness: Observations from experience. Clinical Psychology: Science and Practice, 11, 242-248.

Chambless, D. L., \& Gillis, M. M. (1993). Cognitive therapy for anxiety disorders. Journal of Consulting and Clinical Psychology, 61, 248-260.

Chodron, P. (2001). The places that scare you: A guide to fearlessness in difficult times. Boston, MA: Shambhala Publications.

Craske, M. G., \& Hazlett-Stevens, H. (2002). Facilitating symptom reduction and behavior change in GAD: The issue of control. Clinical Psychology: Science and Practice, 9(1), 69-75.

Crits-Christoph, P., Gibbons, M. B. C., \& Crits-Christoph, K. (2004). Supportive-expressive psychodynamic therapy. In R. G. Heimberg, C. L. Turk, \& D. S. Mennin (Eds.), Generalized anxiety disorder: Advances in research and practice. New York: The Guilford Press.

Dugas, M. J. (2000). Generalized anxiety disorder: So where do we stand? Journal of Anxiety Disorders, $14,31-40$.

Dugas, M. J., Gagnon, F., \& Ladouceur, R. (1998). Generalized anxiety disorder: A preliminary test of a conceptual model. Behavioral Research and Therapy, 36, 215-226.

Easterbrook, J. A. (1959). The effects of emotion on cue utilization and the organization of behavior. Psychological Review, 66, 183-201.

Fairbairn, W. R. D. (1952). An object-relations theory of the personality. New York: Basic Books.

Freeston, M. H., Dugas, M. J., \& Ladouceur, R. (1996). Thoughts, images, worry, and anxiety. Cognitive Therapy and Research, 20, 265-273.

Fromm-Reichmann, F. (1955). Psychiatric aspects of anxiety. In C. M. Thompson, M. Mazer, \& E. Witenberg (Eds.), An outline of psychoanalysis (pp. 113-133). New York: Modern Library.

Geshe, R. (1992). The mind and its functions: A textbook of Buddhist epistemology and psychology. (Stephen Batchelor, Trans \& Ed). Rabten Choeling, Switzerland. Original work published 1978. 
Gould, R. A., Safren, S. A., Washington, D., \& Otto, M. W. (2004). A meta-analytic review of cognitive behavioral treatments. In R. G. Heimberg, C. L. Turk, \& D. S. Mennin (Eds.), Generalized anxiety disorder: Advances in research and practice. New York: The Guilford Press.

Hayes, S. C. (2002). Acceptance, mindfulness, and science. Clinical Psychology: Science and Practice, 9 , 101-106.

Hayes, A. M., \& Feldman, G. (2004). Clarifying the construct of mindfulness in the context of emotion regulation and the process of change in therapy. Clinical Psychology: Science and Practice, 11, 255-262.

Hayes, S. C., Strosahl, K. D., \& Wilson, K. G. (1999). Acceptance and commitment therapy: An experiential approach to behavior change. New York: Guilford.

Horney, K. (1950). Neurosis and human growth: The struggle towards self-realization. New York: Norton.

Jack, B. N., Heller, W., Palmieri, P. A., \& Miller, G. A. (1997). Contrasting patterns of brain activity in anxious apprehension and anxious arousal. Psychophysiology, 36, 628-637. USA: Cambridge University Press.

Kabat-Zinn, J. (1982). An outpatient program in behavioral medicine for chronic pain patients based on the practice of mindfulness meditation: Theoretical considerations and preliminary results. General Hospital Psychiatry, 4, 33-47.

Kabat-Zinn, J. (1990). Full catastrophe living: Using the wisdom of your body and mind to face stress, pain and illness. New York: Delacourt.

Kabat-Zinn, J. (2000). Indra's net at work: The mainstreaming of dharma practice in society. In G. Watson \& S. Batchelor (Eds.), The psychology of awakening: Buddhism, science, and our day-to day lives (pp. 225-249). Nork Beach, ME: Samuel Weiser, Inc.

Kabat-Zinn, J. (2003). Mindfulness-based interventions in context: Past, present, and future. Clinical Psychology: Science and Practice, 10, 144-156.

Kabat-Zinn, J., Massion, A. O., Kristeller, J., Peterson, L. G., Fletcher, K. E., \& Pbert, L. (1992). Effectiveness of a meditation-based stress reduction program in the treatment of anxiety disorders. American Journal of Psychiatry, 149, 936-943.

Kabat-Zinn, J., Wheeler, E., Light, T., Skillings, Z., Scharf, M. J., Cropley, T. G., et al. (1998). Influence of a mindfulness-based stress reduction intervention on rates of skin clearing in patients with moderate to severe psoriasis undergoing phototherapy (UVB) and photochemotherapy (PUVA). Psychosomatic Medicine, 60, 625-632.

Klein, M. (1975). Envy and gratitude and other words 1946-1963. New York: Delacorte Press/Seymour Lawrence.

Koster, E. H. W., Verschuere, B., Geert, C., \& Houwer, J. D. (2004). Selective attention to threat in the dot probe paradigm: Differentiating vigilance and difficulty to disengage. Behavior Research and Therapy, 42, 1183-1192.

Ladouceur, R., Dugas, M. J., Freeston, M. H., Legar, E., Gagnon, F., \& Thibodeau, N. (2000). Efficacy of a new cognitive-behavioral treatment for generalized anxiety disorder: Evaluation in a controlled clinical trial. Journal of Consulting and Clinical Psychology, 68, 957-964.

Leahy, R. L. (2004). Cognitive behavioral therapy. In R. G. Heimberg, C. L. Turk, \& D. S. Mennin (Eds.), Generalized anxiety disorder: Advances in research and practice. New York: The Guilford Press.

Lichtenstein, J., \& Cassidy, J. (1991). The inventory of adult attachment: Validation of a new measure. Paper presented at the biennial meeting of the Society for Research in Child Development, Seattle, WA.

Linehan, M. M. (1993a). Cognitive-behavioral treatment of borderline personality disorder. New York: Guilford.

Linehan, M. M. (1993b). Skills training manual for cognitive behavioral treatment of borderline personality disorder. New York: Guilford.

Martin, J. R. (1997). Mindfulness: A proposed common factor. Journal of Psychotherapy Integration, 7 , 291-312.

Martin, M., Williams, R. M., \& Clark, D. M. (1991). Does anxiety lead to selective processing of threatrelated information? Behavior Research Therapy, 29(2), 147-160.

Matthew, A., \& Mackintosh, B. (1998). A cognitive model of selective processing in anxiety. Cognitive Therapy and Research, 22(6), 539-560.

Mennin, D. S., Heimberg, R. G., \& Turk, C. L. (2004). Clinical presentation and diagnostic features. In R. G. Heimberg, C. L. Turk, \& D. S. Mennin (Eds.), Generalized anxiety disorder: Advances in research and practice. New York: The Guilford Press. 
Miller, J. J., Fletcher, K., \& Kabat-Zinn, J. (1995). Three year follow-up and clinical implications of a mindfulness meditation-based stress reduction intervention in the treatment of anxiety disorders. General Hospital Psychiatry, 17(3), 192-200.

Molina, S., Borkovec, T. D., Peasley, C., \& Person, D. (1998). Content analysis of worrisome streams of consciousness in anxious and dysphoric participants. Cognitive Therapy and Research, 22, 109-123.

Moses, E. B., \& Barlow, D. H. (2006). A new unified treatment approach for emotional disorders based on emotion science. Current Directions in Psychological Science, 15, 146-150.

Newman, M. G., Castonguagy, L. G., \& Borkovec, T. D. (1999). New dimensions in the treatment of generalized anxiety disorder: Interpersonal focus and emotional deepening. Paper presented at the annual meeting of the Society for the Exploration of Psychotherapy Integration, Miami, FL.

Newman, M. G., Castonguay, L. G., Borkovec, T. D., \& Molnar, C. (2004). Integrative therapy for generalized anxiety disorder. In R. G. Heimberg, C. L. Turk, \& D. S. Mennin (Eds.), Generalized anxiety disorder: Advances in research and practice (pp. 320-350). New York: Guilford Press.

Orsillo, S. M., Roemer, L., \& Barlow, D. H. (2003). Integrating acceptance and mindfulness into existing cognitive-behavioral treatment for GAD: A case study. Cognitive and Behavioral Practice, 10, 223-230.

Ost, L. R., \& Breitholtz, E. (2000). Applied relaxation vs. cognitive therapy in the treatment of generalized anxiety disorder. Behavior Research and Therapy, 38, 777-790.

Pabongka, J. (1997). Liberation in the palm of your hand. MA: Wisdom Publication Boston.

Rapgay, L., \& Bystritsky, A. (2008). Introduction to classical mindfulness: Its clinical application. New York: New York Academy of Sciences Special Issue on Mind Body Medicine and Optimal Health.

Roemer, L., \& Orsillo, S. M. (2002). Expanding our conceptualization of and treatment for generalized anxiety disorder: Integrating mindfulness/acceptance-based approaches with existing cognitivebehavioral models. Clinical Psychology: Science and Practice, 9(1), 54-68.

Segal, Z. V., Williams, J. M. G., \& Teasdale, J. D. (2002). Mindfulness-based cognitive therapy for depression: A new approach to prevention relapse. New York: Guilford Press.

Sullivan, H. S. (1953). The interpersonal theory of psychiatry. New York: Norton.

Thera, N. (1973). The heart of Buddhist meditation. New York: Samuel Weiser.

Toneatto, T., \& Nguyen, L. (2007). Does mindfulness meditation improve anxiety and mood symptoms? A review of the controlled research. Canadian Journal of Psychiatry, 52(4), 260-266.

Ustun, T. B., \& Sartorius, N. (Eds.). (1995). Mental illness in general health care: An international study. Chichester, UK: Wiley.

Wells, A. (1995). Meta-cognition and worry: A cognitive model of generalized anxiety disorder. Behavioral and Cognitive Psychotherapy, 23, 301-320.

Wells, A. (2002). GAD, meta-cognition and mindfulness: An information processing analysis. Clinical Psychology: Science and Practice, 9, 95-100.

Wells, A., \& King, P. (2005). Metacognitive therapy for GAD: An open trial. Journal of Behavior Therapy and Experimental Psychiatry, 37(3), 206-212.

Wolpe, J. (1973). The practice of behavioral therapy. New York: Pergamon. 